\title{
RESONANT SPIN-DEPENDENT TUNNELING IN SPIN-VALVE JUNCTIONS IN THE PRESENCE OF PARAMAGNETIC IMPURITIES.
}

\author{
A. Vedyayev ${ }^{1,2}$, D. Bagrets ${ }^{1,2}$, A. Bagrets ${ }^{2}$, and B. Dieny ${ }^{1}$ \\ ${ }^{1}$ CEA/Grenoble, Département de Recherche Fondamentale sur la Matière Condensée, \\ SP2M/NM, 38054 Grenoble, France \\ ${ }^{2}$ Department of Physics, M. V. Lomonosov Moscow State University, 119899 Moscow, Russia
}

The tunnel magnetoresistance (TMR) of F/O/F magnetic junctions, (F's are ferromagnetic layers and $\mathrm{O}$ is an oxide spacer) in the presence of magnetic impurities within the barrier, is investigated. We assume that magnetic couplings exist both between the spin of impurity and the bulk magnetization of the neighboring magnetic electrode, and between the spin of impurity and the spin of tunneling electron. Consequently, the resonance levels of the system formed by a tunneling electron and a paramagnetic impurity with spin $S=1$, are a sextet. As a result the resonant tunneling depends on the direction of the tunneling electron spin. At low temperatures and zero bias voltage the TMR of the considered system may be larger than TMR of the same structure without paramagnetic impurities. It is calculated that an increase in temperature leads to a decrease in the TMR amplitude due to excitation of spin-flip processes resulting in mixing of spin up and down channels. It is also shown that asymmetry in the location of the impurities within the barrier can lead to asymmetry in $I(V)$ characteristics of impurity assisted current and two mechanisms responsible for the origin of this effect are established. The first one is due to the excitation of spin-flip processes at low voltages and the second one arises from the shift of resonant levels inside the insulator layer under high applied voltages.

\section{Introduction}

The observation of the large tunneling magnetoresistance effect at room temperature in tunnel junctions of the form $\mathrm{M} / \mathrm{O} / \mathrm{M}$ ' (where $\mathrm{M}$ and $\mathrm{M}$ ' are magnetic metals and $\mathrm{O}$ is an oxide tunnel barrier) has stimulated a renewed interest for these systems [1, 2, 3]. Besides the fundamental interest for spin-polarized transport, these structures are also foreseen as potential candidates for sensitive magnetic sensors and memory cells in random access memory devices. The first model of spin-dependent tunneling in the framework of classical quantum mechanics was proposed by Słonczewski [4]. However, in this approach no scattering of electrons in the magnetic metallic electrodes was taken into account. This model has been subsequently developed in Refs. [5, 6] by using the Kubo formalism of linear response. The effects of elastic impurity scattering inside the metallic layers and at interfaces between the dielectric and conductive layers could then be incorporated in the model. On the other hand, it is well known [7] that the presence of impurities inside the potential barrier can lead to the mechanism of resonant tunneling when the localized electronic states within the gap of the insulator formed by embedded atoms lie close to the chemical potential of the system. This situation was qualitatively studied in mesoscopic semiconductor system [8] in case of one- and two-impurity resonant channels by means of classical quantum mechanical treatment. The same approach afterwards has been used in [9] with the application to the impurity-assisted tunneling magnetoresistance (TMR). The numerical analysis of this problem which was carried out in Refs. [10, 11] should 
also be mentioned. In paper [9] only the case of spinless impurities was considered, and the author came to the conclusion that the TMR amplitude decreased due to the impurity assisted tunneling. The problem of the paramagnetic impurity assisted tunneling in tunnel magnetic junctions was investigated recently in Ref. [12]. The author claim, that he investigated resonant tunneling through impurity resonance level, however he did not introduce the line-width of this resonance, which, as it will be shown below, does depend on the position of the impurity atom inside the barrier as well on the magnetic configuration of the magnetic layers. As it will be shown below, namely these line-widths define the value of the tunneling conductance and the amplitude of the TMR for spinconserving and spin-flip resonant tunneling. An attempt of the analysis the same problem has also been undertaken in Ref. [13], but nevertheless the microscopic mechanism of electron scattering on the paramagnetic impurity has not been taken into account.

In this paper, we propose a renewed study of the problem of impurity-assisted tunneling in spin-valve junctions of the form $\mathrm{F}_{1} / \mathrm{O} / \mathrm{F}_{3}$, where $\mathrm{F}$ 's are ferromagnetic electrodes and $\mathrm{O}$ is an insulating barrier with embedded paramagnetic impurities, that incorporates the effect of both elastic and non-elastic spin-flip scattering due to the exchange interaction between the itinerant electrons forming the tunneling current and the localized spins of impurities. It will be shown that non-elastic scattering has an essential impact not only on the temperature variation of the TMR (which is a well established result [15]) but also on the $\mathrm{I}-\mathrm{V}$ characteristics of considered structures. The latter effect was predicted in Ref. [15], where the TMR dependence on the electron scattering on interfacial magnons was investigated.

\section{Model}

\subsection{Kubo formula and general expression for the conductivity of the system}

The following simplified model is adopted throughout the paper. First of all, the thickness of an oxide layer is supposed to be much smaller than its in-plane dimension, so that the system may be considered as homogeneous in the $x y$-plane (parallel to the interfaces) and inhomogeneous only in the $z$-direction (growth direction). Within each layer, the electrons are described as a free-electron gas and they undergo scattering on the $3-\mathrm{D} \delta$ function impurity potential within the insulating barrier. Within these approximations, the Hamiltonian of the system has the form

$$
\hat{H}=\hat{H}_{0}+\hat{H}_{\text {int }},
$$

where

$$
\begin{array}{r}
\hat{H}_{0}=-\frac{\hbar^{2}}{2 m(z)} \Delta+U(z)-2 \mu_{B} H_{z}^{\mathrm{eff}}(z)\left(\hat{s}_{z}+\hat{S}_{z}\right) \\
\hat{H}_{\mathrm{int}}=\sum_{i} a_{0}^{3} \delta\left(\mathbf{r}-\mathbf{c}_{i}\right)\left\{\varepsilon_{0}-J(\mathbf{s} \mathbf{S})\right\} .
\end{array}
$$

Here the summation is performed over the location of impurities $\mathbf{c}_{i}$ inside the barrier, $a_{0}$ is the lattice constant, $\varepsilon_{0}$ denotes the scattering potential amplitude on the impurity, $J$ is responsible for the $s-d$ type exchange interaction between a conduction electron spin $\mathbf{s}$ and the impurity spin $\mathbf{S}, U(z)$ is a model step-like potential seen by the conduction electron as it is represented in Fig. 1. We take into account the exchange splitting of 


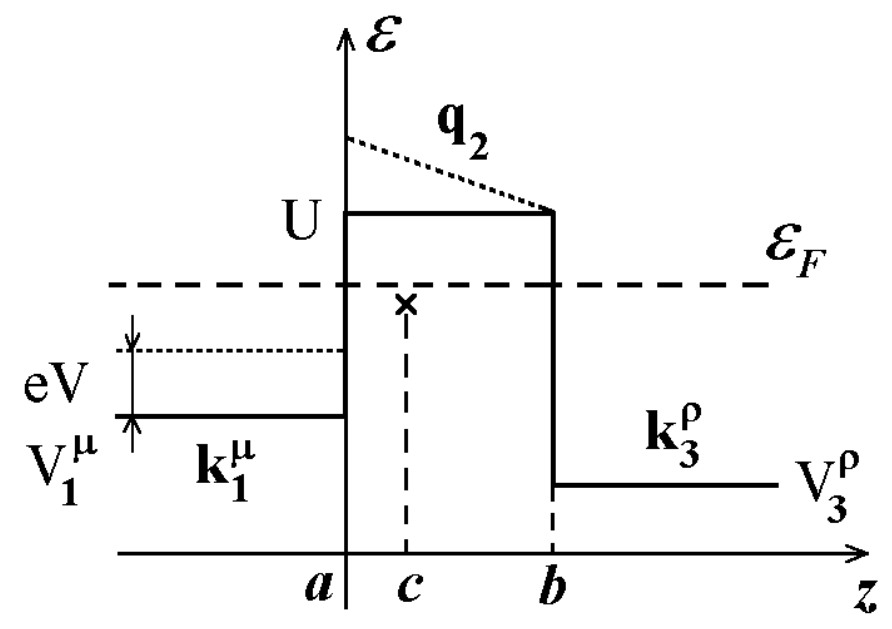

Fig. 1: The potential profile seen by electron propagating through the $\mathrm{F} / \mathrm{O} / \mathrm{F}$ junction comprising impurity defect inside the oxide spacer. $k_{1}^{\mu}, k_{3}^{\rho}, q_{2}$ are the momenta inside the magnetic layers and oxide barrier, respectively. $V_{1(3)}^{\mu(\rho)}$ denotes the spin-dependent conduction band bottom, $U$ is a level of the barrier and $\varepsilon_{F}$ is Fermi energy. The paramagnetic impurity is located at point $c$. The variation of the potential profile under high bias voltage is indicated by the dashed line.

the $d$-band by introducing different values $V_{\mu}^{1,3}$ for the position of the bottom of the conduction band in $\mathrm{F}_{1}$ and $\mathrm{F}_{3}$, depending on the mutual orientation of magnetization in the layers and the spin $\mu=\uparrow, \downarrow$ of the conduction electron. $H_{z}^{\text {eff }}(z)$ represents the effective field acting on impurity and electron spins inside the barrier. The origin of this field is the super-exchange between the spins in the bulk of ferromagnetic layer and in insulating layer. We suppose that $H_{z}^{\text {eff }}(z)$ decreases exponentially with the distance from the interface in the depth of the oxide layer. $m(z)$ corresponds to the effective electron mass that we suppose is equal to $m$ in the ferromagnetic layers and to $m_{0}$ in the insulator. Throughout the paper, it is expressed in units of bare electron mass $m_{e}$. We also assume that the mass of free-like electrons in ferromagnet is slightly differs from $m_{e}$, i.e. $m \approx 1$ and we will eliminate it from all subsequent expressions.

We start from the Keldysh technique for Green functions together with Kubo exact formula of linear response theory for the static conductivity which relates its real part with the current-current correlation function and may be written in the form [16]

$$
\begin{array}{r}
\sigma_{\mu \rho}\left(\mathbf{r}, \mathbf{r}^{\prime}\right)=\frac{1}{2 k_{B} T} \int_{-\infty}^{+\infty}\left\langle j_{\rho}\left(\mathbf{r}^{\prime}, t^{\prime}\right) j_{\mu}(\mathbf{r}, t)\right\rangle d\left(t-t^{\prime}\right) \\
=\frac{1}{2 k_{B} T}\left(\frac{e \hbar}{2 m}\right)^{2} \int_{-\infty}^{+\infty}\left\langle G_{\mu \rho}^{<}\left(\mathbf{r}, t, \mathbf{r}^{\prime}, t^{\prime}\right) \stackrel{\leftrightarrow}{\nabla}_{\mathbf{r}} \stackrel{\leftrightarrow}{\nabla}_{\mathbf{r}^{\prime}} G_{\rho \mu}^{>}\left(\mathbf{r}^{\prime}, t^{\prime}, \mathbf{r}, t\right)\right\rangle d\left(t-t^{\prime}\right)
\end{array}
$$

where $\mu, \rho$ denote the projections of the spin of the electrons, $\overleftrightarrow{\nabla}_{\mathbf{r}}=\frac{1}{2}\left(\vec{\nabla}_{\mathbf{r}}-\overleftarrow{\nabla}_{\mathbf{r}}\right)$ is the asymmetric gradient operator and $G_{\mu \rho}^{<}$and $G_{\rho \mu}^{>}$are corresponding Green functions in Keldysh formalism [17]. $\langle\ldots\rangle$ represents the quantum statistical averaging over the distribution of impurities and degrees of freedom of impurity spin. This expression is most general and holds both for the elastic impurity and defect scattering or inelastic, includ- 
ing magnon and phonon, scattering. To evaluate expression (2) one needs to introduce the retarded Green function $G_{\mu \rho}^{R}\left(z, z^{\prime}, \kappa, \varepsilon\right)$ that in our case is defined by the following differential equation:

$$
\left\{\varepsilon+\frac{\hbar^{2}}{2 m} \frac{\partial^{2}}{\partial z^{2}}-\frac{\kappa^{2}}{2 m}-U(z)\right\} G_{\mu \rho}^{R}\left(z, z^{\prime}, \kappa, \varepsilon\right)=\delta_{\mu \rho} \delta\left(z-z^{\prime}\right)
$$

in the mixed real-space momentum representation [5, 6], where $\kappa=\left(\kappa_{x}, \kappa_{y}\right)$ is the component of the electron momentum in $x y$-plane of the layers and $z$ is the coordinate perpendicular to $x y$-plane. We should note, that by definition the conductivity (2) is defined as a linear response on the externally applied electric field and does not depend on $z$ and $z^{\prime}$ because of the obvious condition $\frac{\partial j(z)}{\partial z}=0$.

Let now denote $k_{1}^{\mu}=\sqrt{2\left(\varepsilon-V_{1}^{\mu}\right)}, k_{3}^{\mu}=\sqrt{2\left(\varepsilon-V_{3}^{\mu}\right)}$ momenta of electrons with energy $\varepsilon$ and spin $\mu$ in the ferromagnetic layers and $q_{0}=\sqrt{2 m_{0}(U-\varepsilon)}$ is an imaginary momentum inside the barrier. By introducing the following functions on $x=\kappa / q_{0}$ :

$$
c_{1}^{\mu}(x)=\sqrt{k_{1}^{\mu 2}-q_{0}^{2} x^{2}}, \quad c_{3}^{\mu}(x)=\sqrt{k_{3}^{\mu 2}-q_{0}^{2} x^{2}}, \quad q_{2}(x)=q_{0} \sqrt{1+x^{2}},
$$

the final expression for the conductance of the system, comprising only one impurity, located at point $\mathbf{c}$, at given temperature $T$ is written as follows:

$$
\sigma(T, \mathbf{c})=\sigma_{0}(T)+\sigma^{\mathrm{imp}}(T, \mathbf{c})
$$

The first term is given by

$$
\sigma_{0}(T)=\frac{q_{0}^{2} e^{2}}{2 \pi \hbar} \sum_{\mu} \int_{-\infty}^{+\infty} d \varepsilon\left(-\frac{\partial f(\varepsilon)}{\partial \varepsilon}\right) \int_{0}^{x_{0}^{\mu}} \frac{x d x}{2 \pi} \frac{16 c_{1}^{\mu} c_{3}^{\mu} m_{0}^{2} q_{2}^{2} e^{-2 q_{2} w}}{\left(m_{0}^{2} c_{1}^{\mu 2}+q_{2}^{2}\right)\left(m_{0}^{2} c_{3}^{\mu 2}+q_{2}^{2}\right)}
$$

where $x_{0}^{\mu}=\min \left\{\frac{k_{1}^{\mu}}{q_{0}}, \frac{k_{3}^{\mu}}{q_{0}}\right\}, f(\varepsilon)=\left[1+e^{\beta\left(\varepsilon-\varepsilon_{F}\right)}\right]^{-1}$ is Fermi function, and $w=b-a$ is the width of the insulating spacer. It represents the well known result for the pure tunneling conductance [4]. The second term $\sigma^{\operatorname{imp}}(T, \mathbf{c})$ is directly related to the impurity assisted tunneling. It is convenient to write it down as a sum of two contributions:

$$
\sigma^{\mathrm{imp}}(T, \mathbf{c})=\sigma_{\mathrm{el}}^{\mathrm{imp}}(T, \mathbf{c})+\sigma_{\mathrm{sf}}^{\mathrm{imp}}(T, \mathbf{c}),
$$

where the first term corresponds to the conductivity due to elastic spin-conserving processes of scattering electron on the impurity and the second one summarizes all other events when the conduction electron changes its spin after tunneling through the barrier. We have derived the analytical expressions for these two terms, which are valid under two assumptions. Namely, under domination of single electron scattering on impurities over multiple scattering of two and more electrons on the same center and under absence of polarization of impurity spin induced by the ejection of spin-polarized electrons. Then the final result for these terms is written as (the details of its derivation are outlined further)

$$
\begin{aligned}
\sigma_{\mathrm{el}}^{\mathrm{imp}}(T, \mathbf{c})=\frac{1}{S}\left(\frac{2 e^{2}}{\pi \hbar}\right) \int_{-\infty}^{+\infty} d \varepsilon\{- & \frac{\partial f_{\uparrow}\left(\varepsilon-\mu_{B} H_{z}^{\mathrm{eff}}\right)}{\partial \varepsilon}\left\langle\left(\hat{t}_{z}^{\uparrow}(\varepsilon)\right)^{\dagger} \hat{t}_{z}^{\uparrow}(\varepsilon)\right\rangle \Phi_{\uparrow}^{L}(\mathbf{c}) \Phi_{\uparrow}^{R}(\mathbf{c})- \\
& \left.\frac{\partial f_{\downarrow}\left(\varepsilon+\mu_{B} H_{z}^{\mathrm{eff}}\right)}{\partial \varepsilon}\left\langle\left(\hat{t}_{z}^{\downarrow}(\varepsilon)\right)^{\dagger} \hat{t}_{z}^{\downarrow}(\varepsilon)\right\rangle \Phi_{\downarrow}^{L}(\mathbf{c}) \Phi_{\downarrow}^{R}(\mathbf{c})\right\},
\end{aligned}
$$




$$
\begin{array}{r}
\sigma_{\mathrm{sf}}^{\mathrm{imp}}(T, \mathbf{c})=\frac{1}{S}\left(\frac{2 e^{2}}{\pi \hbar}\right) \frac{1}{k_{B} T} \int_{-\infty}^{+\infty} d \varepsilon\left\{f_{\uparrow}\left(\varepsilon-\mu_{B} H_{z}^{\mathrm{eff}}\right)\left[1-f_{\downarrow}\left(\varepsilon+\mu_{B} H_{z}^{\mathrm{eff}}\right)\right]\left\langle\hat{t}_{-}(\varepsilon) \hat{t}_{+}(\varepsilon)\right\rangle+\right. \\
\left.f_{\downarrow}\left(\varepsilon+\mu_{B} H_{z}^{\mathrm{eff}}\right)\left[1-f_{\uparrow}\left(\varepsilon-\mu_{B} H_{z}^{\mathrm{eff}}\right)\right]\left\langle\hat{t}_{+}(\varepsilon) \hat{t}_{-}(\varepsilon)\right\rangle\right\} \times \frac{1}{2}\left\{\Phi_{\uparrow}^{L}(\mathbf{c}) \Phi_{\downarrow}^{R}(\mathbf{c})+\Phi_{\downarrow}^{L}(\mathbf{c}) \Phi_{\uparrow}^{R}(\mathbf{c})\right\} .
\end{array}
$$

Here $S$ is the junction area, $\Phi_{\uparrow(\downarrow)}^{L}(\mathbf{c})$ and $\Phi_{\uparrow(\downarrow)}^{R}(\mathbf{c})$ are the probabilities of tunneling of the electron from the left or from the right electrode to impurity, located at point c. Omitting the exponentially small terms, the expression for these probabilities can be written as

$$
\begin{aligned}
\Phi_{\mu}^{L}(\mathbf{c}) & =\int_{0}^{x_{\max }^{\mu}} \frac{x d x}{2 \pi} \frac{2 c_{1}^{\mu} m_{0}^{2} q_{0}^{2}}{\left(m_{0}^{2} c_{1}^{\mu 2}+q_{2}^{2}\right)} e^{-2 q_{2}(c-a)}, \\
\Phi_{\rho}^{R}(\mathbf{c}) & =\int_{0}^{x_{\max }^{\rho}} \frac{x d x}{2 \pi} \frac{2 c_{3}^{\rho} m_{0}^{2} q_{0}^{2}}{\left(m_{0}^{2} c_{3}^{\rho 2}+q_{2}^{2}\right)} e^{-2 q_{2}(b-c)} .
\end{aligned}
$$

The quantities $\left\langle\left(\hat{t}_{z}^{\uparrow(\downarrow)}\right)^{\dagger} \hat{t}_{z}^{\uparrow(\downarrow)}\right\rangle(\varepsilon)$ and $\left\langle\hat{t}_{-} \hat{t}_{+}\right\rangle(\varepsilon),\left\langle\hat{t}_{+} \hat{t}_{-}\right\rangle(\varepsilon)$ in (雨) represent the scattering amplitudes of electron on the impurity center for the case of spin conserving $(|i n, \uparrow\rangle \rightarrow$ $\mid$ out,$\uparrow\rangle)$ or $\mid$ in,$\downarrow\rangle \rightarrow \mid$ out,$\downarrow\rangle)$ and spin-flip $(|i n, \uparrow\rangle \rightarrow \mid$ out,$\downarrow\rangle$ or $\mid$ in,$\downarrow\rangle \rightarrow|o u t, \uparrow\rangle)$ transitions averaged over the distribution of paramagnetic impurity spin. Here $|i n\rangle$ and $\mid$ out $\rangle$ denote the initial and final states of impurity. Operators $\hat{t}_{z}^{\uparrow(\downarrow)}$ and $\hat{t}_{ \pm}$form a one-center matrix $\hat{t}=\left(\begin{array}{c}\hat{t}_{z}^{\uparrow} \\ \hat{t}_{+} \\ \hat{t}_{+} \hat{t}_{z}^{\downarrow}\end{array}\right)$ in the direct product of the linear subspaces of electron's and impurity's spins and are expressed as

$$
\begin{gathered}
\hat{t}_{z}^{\uparrow(\downarrow)}(\varepsilon)=\frac{1}{1-\hat{V}_{z}^{\uparrow(\downarrow)}(\varepsilon) G_{\uparrow(\downarrow)}(\varepsilon)} \hat{V}_{z}^{\uparrow(\downarrow)}(\varepsilon), \\
\hat{t}_{ \pm}(\varepsilon)=-\frac{1}{1-\hat{V}_{z}^{\downarrow(\uparrow)}(\varepsilon) G_{\downarrow(\uparrow)}(\varepsilon)} \hat{S}_{ \pm} \frac{a_{0}^{3} J / 2}{1-a_{0}^{3}\left(\varepsilon_{0}+\frac{1}{2} J \hat{S}_{z}\right) G_{\uparrow(\downarrow)}(\varepsilon)},
\end{gathered}
$$

where effective potentials $\hat{V}_{z}^{\uparrow(\downarrow)}$ are given by

$$
\hat{V}_{z}^{\uparrow(\downarrow)}(\varepsilon)=a_{0}^{3}\left\{\varepsilon_{0} \mp \frac{1}{2} J \hat{S}_{z}+\frac{1}{4} \hat{S}_{\mp} \frac{a_{0}^{3} G_{\downarrow(\uparrow)}(\varepsilon) J^{2}}{1-a_{0}^{3}\left(\varepsilon_{0} \pm \frac{1}{2} J \hat{S}_{z}\right) G_{\downarrow(\uparrow)}(\varepsilon)} \hat{S}_{ \pm}\right\} .
$$

Here $G_{\uparrow(\downarrow)}(\varepsilon)$ is the electron Green function at point c:

$$
G_{\mu}(\varepsilon, \mathbf{c})=\int_{0}^{\kappa_{\max }} G_{\mu \kappa}(\varepsilon, \mathbf{c}) \frac{\kappa d \kappa}{2 \pi}
$$

where $\kappa_{\max }=\frac{2 \sqrt{\pi}}{a_{0}}$ is a cut-off of in plane momentum that stems from the finite size of Brillouin zone (we substitute the Brillouin zone's projection onto $\left(\kappa_{x}, \kappa_{y}\right)$ plane by the circle of radius $\kappa_{\max }$ of the same square in $\kappa_{\|}$-plane). The real and imaginary part of $G_{\kappa}^{\mu}(\varepsilon, \mathbf{c})(\mu$ is the spin index) in the leading order of magnitude are given by

$$
\begin{gathered}
\operatorname{Re} G_{\kappa}^{\mu}(\varepsilon, \mathbf{c})=-\frac{m_{0}}{q_{2}} \\
\operatorname{Im} G_{\kappa}^{\mu}(\varepsilon, \mathbf{c})=-\left(\Phi_{\mu}^{L}(\mathbf{c})+\Phi_{\mu}^{R}(\mathbf{c})\right) .
\end{gathered}
$$

Let us now explain the derivation of expression (4) and clarify the two assumptions under which this formula is valid. To derive (4) from the starting point (2) one can first 

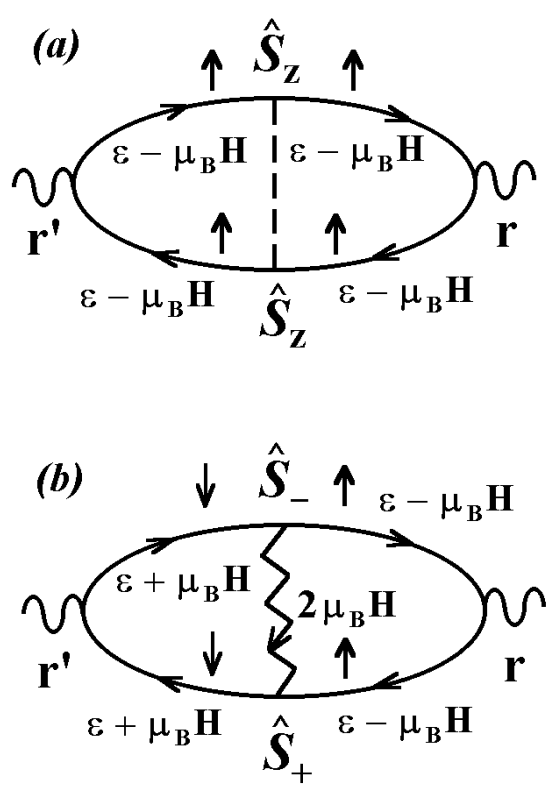

Fig. 2: Two diagrams that make contribution to the conductivity in the second order of perturbation theory in case of $a$ ) elastic spin- conserving scattering and $b$ ) non-elastic spin-flip scattering.

of all examine two diagrams (a) and (b) (see Fig. 2) that contribute to spin-conserving and spin-flip part of $\sigma^{\mathrm{imp}}(T, \mathbf{c})$ at second order of $J$, respectively. One may easy verify that a general structure of these diagrams is just the same as the final result in form (迎) with the mere difference that the one-center $t$-matrix is reduced at first order of $J$ to the initial potential $\varepsilon_{0}-\frac{J}{2}\left(\begin{array}{cc}\hat{S}_{z} & \hat{S}_{-} \\ \hat{S}_{+} & -\hat{S}_{z}\end{array}\right)$.

Moreover, the diagram (b) contains both direct and indirect processes in equal proportion with common factor $1 / 2$ for any of the possible channels $\mid$ in,$\uparrow\rangle \rightarrow \mid$ out, $\downarrow\rangle$ or $|i n, \downarrow\rangle \rightarrow \mid$ out,$\uparrow\rangle$. The thermodynamic averaging $\langle\ldots\rangle$ in the second-order expansion is simply reduced to the averaging over the Boltzman distribution of the impurity spin in the "external" effective magnetic field $H_{z}^{\text {eff }}$ which was introduced in (1), i.e. with the density matrix $\hat{\rho}_{0}=Z^{-1} \exp \left\{\frac{2 \mu_{B} H_{z}^{\mathrm{eff}} \hat{S}_{z}}{k_{B} T}\right\}$, where $Z=2 \cosh \left(\frac{2 \mu_{B} H_{z}^{\mathrm{eff}}}{k_{B} T}\right)+1$. After that, it is easy to check that the total probabilities (with account of Fermi factors of electron states) of direct and inverse processes are equal which means that the principle of detailed equilibrium holds. In particular it leads to the vanishing of spin-flip processes in a system at zero temperature and vanishing voltage bias.

After that preliminary discussion two assumptions should be made to justify the result (州):

i) We assume that the occupation of the given impurity center simultaneously by two electrons with different spin (due to Pauli principle) is a rather rare event or, in other words, we do not take into account many-electrons effects. It may be justified: a) by Coulomb interaction between electrons that make unprofitable their arrangement at the same site of the lattice; b) by the large number of impurity centers that provides a sufficiently large number of one-step channels so that electrons may be considered as 
independent.

ii) We also neglect the influence of electron current on the statistical distribution of paramagnetic spins inside the oxide barrier. This assumption is valid for practical intensity of tunneling current which is low enough not to produce a spin polarization of impurities by injection of spin-polarized charge carriers.

Under these assumptions the expression (4) can be obtained by simple substitution of scattering potential $\hat{H}_{\text {int }}$ at site $\mathbf{c}_{i}$ on Fig. 2 by the corresponding one-center $t$-matrix in accordance with (5) and assuming that the averaging over the degrees of freedoms of the impurity is carried out by means of unperturbed density matrix $\hat{\rho}_{0}=Z^{-1} \exp \left\{\frac{2 \mu_{B} H_{z}^{\text {eff }} \hat{S}_{z}}{k_{B} T}\right\}$. In this form the structure of the result (我) is similar to the one obtained in Ref. [15], where the spin-flip scattering of electrons at interfaces of tunnel junctions was investigated in the framework of tunneling Hamiltonian and the second order perturbation theory.

\subsection{Resonant tunneling in the case of nonmagnetic impurities}

To extract the physical nature of resonant tunneling through the impurity states contained in expression (4) we proceed as follows. For the sake of clarity and simplicity we consider first the case of zero-spin impurity. Then only one element of $t$-matrix at cite $\mathbf{c}$ survives

$$
t_{0}^{\uparrow(\downarrow)}(\varepsilon)=\frac{a_{0}^{3} \varepsilon_{0}}{1-a_{0}^{3} \varepsilon_{0} G_{\uparrow(\downarrow)}(\varepsilon)} .
$$

It defines the position of a resonant level inside the gap of the dielectric band structure by finding the root of the equation $a_{0}^{3} \varepsilon_{0} \operatorname{Re} G_{\uparrow(\downarrow)}\left(\varepsilon_{i}\right)=1$. From expression (6), it follows that the real part of the Green function $\operatorname{Re} G_{\mu}(\varepsilon, \mathbf{c})$ is independent on $\mathbf{c}$ and spin $\mu$ up to exponentially small terms. Therefore, the position of level $\varepsilon_{i}$ is weakly depend both on the position of impurity inside the barrier and on the direction of the spin of tunneling electron. Evidently, only those impurities for which $\varepsilon_{i}$ is close to the chemical potential $\varepsilon_{F}$ contribute to a significant extent of the total current at low bias voltage. Therefore, it is possible to expand the denominator in $t_{0}^{\uparrow(\downarrow)}(\varepsilon)$ in powers of $\left(\varepsilon_{i}-\varepsilon\right)$. If we now introduce the position dependent line-widths

$$
\Gamma_{\mu}^{L}(\mathbf{c})=\Phi_{\mu}^{L}(\mathbf{c}) / \operatorname{Re} G^{\prime}\left(\varepsilon_{F}\right), \quad \Gamma_{\mu}^{R}(\mathbf{c})=\Phi_{\mu}^{R}(\mathbf{c}) / \operatorname{Re} G^{\prime}\left(\varepsilon_{F}\right),
$$

where $\operatorname{Re} G^{\prime}\left(\varepsilon_{F}\right)=\left.\frac{\partial}{\partial \varepsilon} \operatorname{Re} G(\varepsilon)\right|_{\varepsilon=\varepsilon_{F}}$ is the energy derivative of the electron Green function at Fermi level, then we obtain the general formula for the resonant case of impurity assisted tunneling

$$
\sigma^{\operatorname{imp}}(\mathbf{c}) \simeq \frac{1}{S}\left(\frac{2 e^{2}}{\pi \hbar}\right) \sum_{\mu} \int_{-\infty}^{+\infty} \sum_{i} \frac{\Gamma_{\mu}^{L}(\mathbf{c}) \Gamma_{\mu}^{R}(\mathbf{c})}{\left(\varepsilon_{F}-\varepsilon_{i}\right)^{2}+\Gamma_{\mu}^{2}(\mathbf{c})}\left(-\frac{\partial f(\varepsilon)}{\partial \varepsilon}\right) d \varepsilon
$$

where $\Gamma_{\mu}(\mathbf{c})=\Gamma_{\mu}^{L}(\mathbf{c})+\Gamma_{\mu}^{R}(\mathbf{c})$ and the summation by $i$ is performed over all resonant levels. For the qualitative analysis, one may evaluate the expressions (7) for $\Gamma_{\mu}^{R(L)}(\mathbf{c})$ approximately by considering the case $\kappa=0$ which is valid if $e^{-2 q_{0} w} \ll 1$. In this approximation

$$
\begin{aligned}
\Gamma_{\mu}^{L}(\mathbf{c}) & =\frac{2 k_{1 \mu}^{F} m_{0}}{m_{0}^{2} k_{1 \mu}^{F}+q_{0}^{2}}\left(\frac{q_{0}^{2}}{2 m_{0}}\right) \frac{e^{-2 q_{0}(c-a)}}{c-a} \\
\Gamma_{\mu}^{R}(\mathbf{c}) & =\frac{2 k_{3 \mu}^{F} m_{0}}{m_{0}^{2} k_{3 \mu}^{F}+q_{0}^{2}}\left(\frac{q_{0}^{2}}{2 m_{0}}\right) \frac{e^{-2 q_{0}(b-c)}}{b-c}
\end{aligned}
$$


and expression (8) reproduces the result of Ref. [8]. To proceed further, we discuss some assumptions concerning the parameters of the model. We consider the case of Co electrode and $\mathrm{Al}_{2} \mathrm{O}_{3}$ as an oxide layer and take typical values of $k_{\uparrow}^{F}=1.09 \AA^{-1}, k_{\downarrow}^{F}=0.42 \AA^{-1}$, $m \approx 1$ for itinerant electrons in Co and a typical barrier height for $\mathrm{Al}_{2} \mathrm{O}_{3}$ (measured from the Fermi level $\varepsilon_{F}$ ) $U_{0}-\varepsilon_{F}=3 \mathrm{eV}$ with an effective mass $m_{0}=0.4$ (Ref. [9]), that gives $q_{0} \simeq 0.56 \AA^{-1}$. Assuming the thickness of the barrier $w \simeq 20 \AA^{-1}$, one may estimate the conductance $\sigma_{0}$ of the system without impurity from (3) by means of approximate formula

$$
\sigma_{0} \simeq \frac{2 e^{2}}{\pi^{2} \hbar}\left(\frac{q_{0}}{w}\right) \sum_{\mu} \frac{k_{1 \mu}^{F} k_{3 \mu}^{F} q_{0}^{2} m_{0}^{2} e^{-2 q_{0} w}}{\left(m_{0}^{2} k_{1 \mu}^{F 2}+q_{0}^{2}\right)\left(m_{0}^{2} k_{3 \mu}^{F 2}+q_{0}^{2}\right)},
$$

that leads to $\mathrm{GMR} \simeq 16 \%$. To estimate the value of the line-width (9) we consider impurities located close to left interface at a distance, say, of two atomic layers which corresponds to $(c-a) \simeq 4 \AA$. For spin up electrons it gives $\Gamma_{\uparrow}(\mathbf{c}) \simeq 0.02 \mathrm{eV}$. Further in this paper we restrict ourselves to the case of temperature interval from $4.2-300 \mathrm{~K}$ $(0.025 \mathrm{eV})$. We assume that the impurity levels $\varepsilon_{i}$ in the band gap of the insulator form a narrow impurity band of width $\Delta \varepsilon$ which spreads symmetrically with respect to Fermi level $\varepsilon_{F}$ and, following Ref. [8], we introduce its density of states $\nu(\varepsilon)$ per unit volume and unit energy interval. We assume that $\Delta \varepsilon$ is of the order 0.1 to $0.2 \mathrm{eV}$, i.e. an order of magnitude greater that the above estimated line-width. In this context, with a good accuracy, the impurity conductance (8) rewrites as follows:

$$
\sigma^{\operatorname{imp}}(\mathbf{c}) \simeq \frac{2 e^{2}}{\hbar} \nu\left(\varepsilon_{F}\right) \sum_{\mu} \int_{-\infty}^{+\infty}\left(-\frac{\partial f}{\partial \varepsilon}\right) \frac{\Gamma_{\mu}^{L}(\mathbf{c}) \Gamma_{\mu}^{R}(\mathbf{c})}{\Gamma_{\mu}(\mathbf{c})} \rho(\varepsilon, \mathbf{c}) d \varepsilon,
$$

where factor

$$
\rho(\varepsilon, \mathbf{c})=\frac{2}{\pi} \arctan \left(\frac{\Delta \varepsilon}{2 \Gamma_{\mu}(\mathbf{c})}\right)
$$

arises from the integration of exp. (8) over impurity levels $\varepsilon_{i}$ in the range of impurity band. Due to above mentioned estimations equality $\rho(\varepsilon, \mathbf{c}) \simeq 1$ holds with a good degree of accuracy and in this case exp. (11) becomes in agreement with Refs. [8, 9].

\subsection{Resonant tunneling in the case of paramagnetic impurities}

To investigate the general case of paramagnetic impurity we follow the same procedure as in the previous section. Let $\mathbf{J}=\mathbf{s}+\mathbf{S}$ be the total magnetic moment of the system. We may state that $\left[H, J_{z}\right]=0$ and, therefore, $J_{z}$ is a good quantum number. We regard the $\hat{t}$-matrix (5) as an operator acting on the spinor subspace $|\sigma, m\rangle$, where $\sigma= \pm \frac{1}{2}$ and $m= \pm 1,0$ corresponds to the projection of the $z$-component of electron and impurity spin, respectively (we consider the case $\mathbf{S}=1$ ). As far as its total magnetic moment along the $z$-axis $J_{z}=s_{z}+S_{z}$ is conserved, the matrix elements $\left\langle\sigma_{1} m_{1}|\hat{t}| \sigma_{2} m_{2}\right\rangle$ are nonzero only if $m_{1}+\sigma_{1}=m_{2}+\sigma_{2}$ and, therefore, it is convenient to introduce the notation $t_{m_{j}}^{\sigma_{1} \sigma_{2}}=\left\langle\sigma_{1} m_{1}|\hat{t}| \sigma_{2} m_{2}\right\rangle$, where $m_{j}=m_{1}+\sigma_{1}=m_{2}+\sigma_{2}$. These elements are simply calculated from (5). The non-zero ones are written as follows

$$
\begin{aligned}
t_{3 / 2}^{\uparrow \uparrow} & =\frac{a_{0}^{3}\left(\varepsilon_{0}-J / 2\right)}{1-a_{0}^{3}\left(\varepsilon_{0}-J / 2\right) G_{\uparrow}(\varepsilon)}, \\
t_{-3 / 2}^{\downarrow \downarrow} & =\frac{a_{0}^{3}\left(\varepsilon_{0}-J / 2\right)}{1-a_{0}^{3}\left(\varepsilon_{0}-J / 2\right) G_{\downarrow}(\varepsilon)},
\end{aligned}
$$


and $\hat{t}_{ \pm 1 / 2}=\left(\begin{array}{cc}t_{ \pm 1 / 2}^{\downarrow \downarrow} & t_{ \pm 1 / 2}^{\downarrow \uparrow} \\ t_{ \pm 1 / 2}^{\uparrow \downarrow} & t_{ \pm 1 / 2}^{\uparrow \uparrow}\end{array}\right)$ corresponding to the subspace $m_{j}= \pm \frac{1}{2}$ with

$$
\begin{aligned}
t_{1 / 2}^{\downarrow \downarrow}\left(t_{-1 / 2}^{\uparrow \uparrow}\right) & =\frac{a_{0}^{3}}{\Delta_{ \pm 1 / 2}(\varepsilon)}\left\{\varepsilon_{0}+J / 2-a_{0}^{3} G_{\uparrow(\downarrow)}(\varepsilon)\left(\varepsilon_{0}-J / 2\right)\left(\varepsilon_{0}+J\right)\right\}, \\
t_{1 / 2}^{\uparrow \uparrow}\left(t_{-1 / 2}^{\downarrow \downarrow}\right) & =\frac{a_{0}^{3}}{\Delta_{ \pm 1 / 2}(\varepsilon)}\left\{\varepsilon_{0}-a_{0}^{3} G_{\downarrow(\uparrow)}(\varepsilon)\left(\varepsilon_{0}-J / 2\right)\left(\varepsilon_{0}+J\right)\right\} \\
t_{ \pm 1 / 2}^{\uparrow \downarrow} & =t_{ \pm 1 / 2}^{\downarrow \uparrow}=-\frac{a_{0}^{3}}{\sqrt{2} \Delta_{ \pm 1 / 2}} J
\end{aligned}
$$

where denominators are

$$
\Delta_{ \pm 1 / 2}(\varepsilon)=\left(1-a_{0}^{3} G_{\downarrow}(\varepsilon)\left(\varepsilon_{0}-J / 2\right)\right)\left(1-a_{0}^{3} G_{\uparrow}(\varepsilon)\left(\varepsilon_{0}+J\right)\right) \pm a_{0}^{3} J\left(G_{\uparrow}(\varepsilon)-G_{\downarrow}(\varepsilon)\right) .
$$

As can be seen, two poles of the $\hat{t}$-matrix defined from equations $a_{0}^{3} \operatorname{Re} G\left(\varepsilon_{3 / 2}\right)\left(\varepsilon_{0}-J / 2\right)=$ 1 and $a_{0}^{3} \operatorname{Re} G\left(\varepsilon_{1 / 2}\right)\left(\varepsilon_{0}+J\right)=1$ correspond to two multiplets $\varepsilon_{3 / 2}$ and $\varepsilon_{1 / 2}$ with a total angular momentum $j=3 / 2$ and $j=1 / 2$, respectively. If $J>0$, then $\varepsilon_{3 / 2}<\varepsilon_{1 / 2}$, i.e. the multiplet with $j=3 / 2$ has a lower energy than one with $j=1 / 2$. As for non-magnetic impurity, we restrict ourselves by considering the regime of only one-channel resonant tunneling. We assume that $J>0$ and the lowest impurity levels $\varepsilon_{i}=\varepsilon_{3 / 2}$ corresponding to the multiplet with $j=3 / 2$ lie close to $\varepsilon_{F}$. We note that the typical value of exchange coupling $J$ is of order $1 \mathrm{eV}$ and due to this fact we may eliminate the resonant level $\varepsilon_{1 / 2}$ from further consideration. Then, as in the previous analysis for a non- magnetic impurity, only the resonant part of the $\hat{t}$ - matrix $(12,13)$ at energies close to chosen $\varepsilon_{3 / 2}$ is essential for the subsequent calculations. Expressions (12,13) can be easily written as follows:

$$
\begin{gathered}
t_{ \pm 3 / 2}^{\uparrow \uparrow(\downarrow)}(\varepsilon)=\frac{1}{G^{\prime}(\varepsilon)} \frac{1}{\varepsilon-\varepsilon_{i}+i \Gamma_{\uparrow(\downarrow)}(\mathbf{c})} \\
\hat{t}_{1 / 2}(\varepsilon)=\frac{1}{G^{\prime}(\varepsilon)} \frac{1}{\varepsilon-\varepsilon_{i}+i \gamma_{\uparrow}(\mathbf{c})}\left(\begin{array}{cc}
\frac{1}{3} & \frac{\sqrt{2}}{3} \\
\frac{\sqrt{2}}{3} & \frac{2}{3}
\end{array}\right), \\
\hat{t}_{-1 / 2}(\varepsilon)=\frac{1}{G^{\prime}(\varepsilon)} \frac{1}{\varepsilon-\varepsilon_{i}+i \gamma_{\downarrow}(\mathbf{c})}\left(\begin{array}{cc}
\frac{2}{3} & \frac{\sqrt{2}}{3} \\
\frac{\sqrt{2}}{3} & \frac{1}{3}
\end{array}\right),
\end{gathered}
$$

where $\gamma_{\uparrow}(\mathbf{c})=\frac{2}{3} \Gamma_{\uparrow}(\mathbf{c})+\frac{1}{3} \Gamma_{\downarrow}(\mathbf{c}), \gamma_{\downarrow}(\mathbf{c})=\frac{1}{3} \Gamma_{\uparrow}(\mathbf{c})+\frac{2}{3} \Gamma_{\downarrow}(\mathbf{c})$ are the inverse lifetimes of the resonant states with $m_{j}= \pm 1 / 2$. This result allows simple qualitative interpretation. Let us look, for a example, at quantum states with $m_{j}=1 / 2$. From elementary quantum mechanical theory one may conclude that

$$
\begin{gathered}
\phi_{1 / 2}^{\uparrow}=\left|\uparrow, m_{s}=0\right\rangle=\sqrt{\frac{2}{3}}\left|j=\frac{3}{2}, m_{j}=\frac{1}{2}\right\rangle+\sqrt{\frac{1}{3}}\left|j=\frac{1}{2}, m_{j}=\frac{1}{2}\right\rangle, \\
\phi_{1 / 2}^{\downarrow}=\left|\downarrow, m_{s}=1\right\rangle=\sqrt{\frac{1}{3}}\left|j=\frac{3}{2}, m_{j}=\frac{1}{2}\right\rangle-\sqrt{\frac{2}{3}}\left|j=\frac{1}{2}, m_{j}=\frac{1}{2}\right\rangle .
\end{gathered}
$$

As we have assumed, only $\left|j=\frac{3}{2}, m_{j}=\frac{1}{2}\right\rangle$ gets into resonance and, therefore, e.g. $t_{1 / 2}^{\uparrow \downarrow} \sim\left\langle\phi_{1 / 2}^{\uparrow}|\hat{t}| \phi_{1 / 2}^{\downarrow}\right\rangle \sim \sqrt{2} / 3$ in agreement with (14). On the other hand,

$$
\left|j=\frac{3}{2}, m_{j}=\frac{1}{2}\right\rangle=\sqrt{\frac{2}{3}}\left|\uparrow, m_{s}=0\right\rangle+\sqrt{\frac{1}{3}}\left|\downarrow, m_{s}=1\right\rangle
$$


and, hence, its inverse life time is given by $\tau_{1 / 2}^{-1}=\frac{2}{3} \tau_{\uparrow}^{-1}+\frac{1}{3} \tau_{\downarrow}^{-1}$.

We substitute all $\hat{t}$-matrix elements in (四) by its resonance expansion (14). To proceed further, one has to perform in (身) the configuration averaging over all impurity centers and thermodynamic one over all possible channels. Suppose, that the impurities are distributed uniformly in the space in the interval $\left[z_{0}-\Delta, z_{0}+\Delta\right]$ along $z$-direction with the width of $2 \Delta$ and center $z_{0}$ which we have chosen to be close to left (L) ferromagnetic contact. After that, it is possible, first of all, to average the Lorentzian peaks over the distribution of impurity levels by averaging them over $\varepsilon_{i}$ in the range of impurity band with factor $\nu\left(\varepsilon_{F}\right)$ and to perform the thermodynamic averaging by integrating over $\varepsilon$ and neglecting the dependencies of $\Gamma^{L(R)}(\mathbf{c})$ on energy. On the third step, the averaging over the space distribution of impurities along $z$-direction should be made. Following the outlined procedure, the total conductance (4) as a function of temperature for the parallel and antiparallel alignment of magnetization of the ferromagnetic layers is written as a sum of factorized terms over all possible scattering channels:

$$
\begin{gathered}
\sigma^{P}(T)=\frac{2 e^{2}}{\hbar} \sum_{\mu \rho m_{j}} P_{m_{j}}^{\mu \rho}\left(\frac{\mu_{B} H_{z}^{\mathrm{eff}}}{k_{B} T}\right){ }^{P} \sigma_{m_{j}}^{\mu \rho}\left(z_{0}, \Delta\right) \nu\left(\varepsilon_{F}\right)+\sigma_{0}^{P}, \\
\sigma^{A P}(T)=\frac{2 e^{2}}{\hbar} \sum_{\mu \rho m_{j}} P_{m_{j}}^{\mu \rho}\left(\frac{\mu_{B} H_{z}^{\mathrm{eff}}}{k_{B} T}\right){ }^{A P} \sigma_{m_{j}}^{\mu \rho}\left(z_{0}, \Delta\right) \nu\left(\varepsilon_{F}\right)+\sigma_{0}^{A P},
\end{gathered}
$$

where

$$
\sigma_{m_{j}}^{\mu \rho}\left(z_{0}, \Delta\right)=\frac{1}{2 \Delta} \int_{z_{0}-\Delta}^{z_{0}+\Delta} \sigma_{m_{j}}^{\mu \rho}(c) \rho\left(\varepsilon_{F}, c\right) d c .
$$

The origine of $\rho\left(\varepsilon_{F}, c\right)$ is the same as in exp. (11) and explicit form of functions $P_{m_{j}}^{\mu \rho}(h)$ and $\sigma_{m_{j}}^{\mu \rho}(c)$ is given in Appendix A. We have also used the same notation of matrix indexes as it was previously introduced for $\hat{t}$-matrix elements. $\sigma_{0}^{P}$ and $\sigma_{0}^{A P}$ are the tunnel conductances of the pure system in accordance with (3). Factors $P_{m_{j}}^{\mu \rho}$ and $\sigma_{m_{j}}^{\mu \rho}$ represent the thermodynamic and quantum mechanical probabilities of the given process, respectively. Expressions (16) are the final results of this section and their analysis is presented below (see Sec. III).

\subsection{Dependence of conductivity on bias-voltage}

We are also interested in $I(V)$ characteristic of the considered system. To derive the

general formula for the current, one may simply extend the expressions (团) to the case of finite applied bias voltage. Consider, for example, the contribution to the total current $I$, coming from all possible channels of the form $|i n, \uparrow\rangle \rightarrow|o u t, \downarrow\rangle$ for tunnel electrons moving from the left electrode to the right electrode and of the form $|i n, \downarrow\rangle \rightarrow \mid$ out, $\uparrow\rangle$ for electrons moving from the right to the left, respectively, i.e. in both cases an electron has an "up" projection of spin in the left contact and "down" projection of spin in the right one after or before scattering. From the general concept, one may conclude that this contribution to the current can be written as

$$
\begin{array}{r}
I^{\uparrow \downarrow}(V)=\frac{1}{S}\left(\frac{2 e^{2}}{\pi \hbar}\right) \int_{-\infty}^{+\infty} d \varepsilon\left\{f_{\uparrow}\left(\varepsilon-\mu_{B} H_{z}^{\mathrm{eff}}-e V\right)\left[1-f_{\downarrow}\left(\varepsilon+\mu_{B} H_{z}^{\mathrm{eff}}\right)\right]\left\langle\hat{t}_{-}(\varepsilon) \hat{t}_{+}(\varepsilon)\right\rangle-\right. \\
\left.f_{\downarrow}\left(\varepsilon+\mu_{B} H_{z}^{\mathrm{eff}}\right)\left[1-f_{\uparrow}\left(\varepsilon-\mu_{B} H_{z}^{\mathrm{eff}}-e V\right)\right]\left\langle\hat{t}_{+}(\varepsilon) \hat{t}_{-}(\varepsilon)\right\rangle\right\} \Phi_{\uparrow}^{L}(\mathbf{c}) \Phi_{\downarrow}^{R}(\mathbf{c}),(17
\end{array}
$$


where it is assumed that the voltage bias is applied from the left to the right direction. It is important to notice that inelastic spin-flip processes of the electron scattering on the impurity were taken into account in derivation of the exp. (17) but they were omitted in Ref. [12]. Analogous expressions can be written for all other channels. In the case under consideration, expression (17) contains two regimes of non-linear behavior of $I(V)$ characteristic. The first one reproduces a zero bias anomaly due to excitation of spinflip processes at low bias voltages of order of magnitude $\mu_{B} H_{z}^{\text {eff }}$ (we believe that it is of order $5 \mathrm{mV}$ ). In this range, as before, one may assume that the resonance amplitudes $\left\langle\left(\hat{t}_{z}^{\uparrow \downarrow}\right)^{\dagger}\left(\hat{t}_{z}^{\uparrow(\downarrow)}\right)\right\rangle$ and $\left\langle\hat{t}_{-} \hat{t}_{+}\right\rangle$are nearly independent of the energy after averaging over all possible configurations of impurities. As a result, the voltage dependence of total currents for parallel and antiparallel configurations are given by formulae similar to (16):

$$
\begin{aligned}
I^{P}(V, T) & =\frac{2 e}{\hbar} \sum_{\mu \rho m_{j}} I_{m_{j}}^{\mu \rho}\left(V, H_{z}^{\mathrm{eff}}\right)^{P} \sigma_{m_{j}}^{\mu \rho}\left(z_{0}, \Delta\right) \nu\left(\varepsilon_{F}\right)+\sigma_{0}^{P} V \\
I^{A P}(V, T) & =\frac{2 e}{\hbar} \sum_{\mu \rho m_{j}} I_{m_{j}}^{\mu \rho}\left(V, H_{z}^{\mathrm{eff}}\right){ }^{A P} \sigma_{m_{j}}^{\mu \rho}\left(z_{0}, \Delta\right) \nu\left(\varepsilon_{F}\right)+\sigma_{0}^{A P} V .
\end{aligned}
$$

The expressions for $I_{m_{j}}^{\mu \rho}\left(V, H_{z}^{\text {eff }}\right)$ are given in Appendix B. The voltage dependent conductances $\sigma^{P}(V, T)$ and $\sigma^{A P}(V, T)$ can be obtained from (17) by derivation with respect to $V$. The detailed analysis of this physical situation is presented in the next section.

The second source of possible non-linear character of $I(V)$ dependence is the variation of potential profile $U(z)$ (see Fig. 1) under applied bias voltage. It follows, then, that the latter introduces the correction to exp. (9) and (10) and they can be calculated with the use of Wentzel-Kramers-Brillouin (WKB) approximation [18] assuming that the applied voltage produces the uniform electrical field inside the insulating layer. In the case of pure tunnel conductance it is known[19] that both conductances for the parallel and antiparallel configurations increase with the increase of applied voltage so that the TMR as a function of $V$, defined as $\frac{I^{P}(V)-I^{A P}(V)}{I^{A P}(V)}$, drops significantly at the voltages of order $1 \mathrm{eV}$. The contribution of impurity assisted tunneling may change considerably this situation in the case of non-uniform spatial distribution of impurities, e.g. when they are distributed in the vicinity of only one electrode. In this particular situation, as we will show, the essential variation of TMR amplitude in the case of magnetic impurities (in contrast to non-magnetic ones) will take place at the bias voltages compared with impurity band width $\Delta \varepsilon$.

For the sake of simplicity we consider, first, the case of non-magnetic impurities. In the WKB approximation the contribution from all impurities, located at given point $\mathbf{c}$, to the total current $I(V)$ will have the form similar to (9) and (11):

$$
j^{\operatorname{imp}}(\mathbf{c})=\frac{2 e^{2}}{\hbar} \nu\left(\varepsilon_{F}\right) \sum_{\mu} \int_{-\infty}^{+\infty}\{f(\varepsilon-e V)-f(\varepsilon)\} \frac{\Gamma_{\mu}^{L}(\mathbf{c}) \Gamma_{\mu}^{R}(\mathbf{c})}{\Gamma_{\mu}^{L}(\mathbf{c})+\Gamma_{\mu}^{R}(\mathbf{c})} \rho(\varepsilon, V) d \varepsilon,
$$

where

$$
\begin{aligned}
\Gamma_{\mu}^{L}(\mathbf{c}) & =\frac{k_{1 \mu}^{F} q_{a} m_{0} \tau_{a}^{-1}}{\left(q_{a}^{-}\right)^{2}+k_{1 \mu}^{F 2} m_{0}^{2}} e^{-S_{a} / \hbar} \\
\Gamma_{\mu}^{R}(\mathbf{c}) & =\frac{k_{3 \mu}^{F} q_{b} m_{0} \tau_{b}^{-1}}{\left(q_{b}^{+}\right)^{2}+k_{3 \mu}^{F 2} m_{0}^{2}} e^{-S_{b} / \hbar} .
\end{aligned}
$$


Here $q_{b}^{2}=q_{0}^{2}=2 m_{0}(U-\varepsilon), q_{a}^{2}=q_{0}^{2}+2 m_{0} e V$ are imaginary momenta of electron with the energy $\varepsilon$ in the vicinity of the right and the left electrode, $q_{a(b)}^{ \pm}=q_{0} \pm \frac{1}{2} \frac{e E m_{0}}{q_{a(b)}^{2}}, E$ is the electric field in the device. We also introduce $q_{c}=q_{0}+2 m_{0} e V(b-c) / w$ imaginary momenta of electron on the impurity center. Then $S_{a}=\frac{q_{a}^{3}-q_{c}^{3}}{3 m_{0} e E}, S_{b}=\frac{q_{c}^{3}-q_{b}^{3}}{3 m_{0} e E}$ represent the classical actions along the path from the left contact to the point $\mathbf{c}$ in the barrier and, afterwards, from this point to the right contact, respectively; $\tau_{a}=\frac{q_{a}-q_{c}}{e E}$ and $\tau_{b}=\frac{q_{c}-q_{b}}{e E}$ denote the passage times associated with these paths. Factor

$$
\rho(\varepsilon, V)=\frac{1}{\pi}\left\{\arctan \left[\frac{\varepsilon-\varepsilon_{F}-e V\left(\frac{b-c}{w}\right)+\frac{\Delta \varepsilon}{2}}{\Gamma_{\mu}^{L}(\mathbf{c})+\Gamma_{\mu}^{R}(\mathbf{c})}\right]-\arctan \left[\frac{\varepsilon-\varepsilon_{F}-e V\left(\frac{b-c}{w}\right)-\frac{\Delta \varepsilon}{2}}{\Gamma_{\mu}^{L}(\mathbf{c})+\Gamma_{\mu}^{R}(\mathbf{c})}\right]\right\}
$$

as before, arises from the summation over all impurity levels $\varepsilon_{i}$ and gives the relative weight of all resonant channels with energy $\varepsilon$. To clarify the situation, it is sufficiently to consider the most resonant channel with energy $\varepsilon_{r}=\varepsilon_{F}+e V(b-c) / w$ at which $\rho(\varepsilon, V)$ reaches its maximum. One may note that $\varepsilon_{r}$ corresponds to the resonant impurity level that exactly coincides with Fermi energy at vanishing voltage and it shifts linearly with the increase of applied bias depending on the position $\mathbf{c}$ of impurity inside the barrier. As it was stated earlier, the most interesting case takes place when the point $\mathbf{c}$ is situated close to the left contact. Then one can see that $\Gamma^{L}(\mathbf{c}) \gg \Gamma^{R}(\mathbf{c})$ and, thus, $j^{\text {imp }}(\mathbf{c}) \sim \Gamma^{R}(\mathbf{c}) \rho\left(\varepsilon_{r}, V\right)$. At bias voltages much more lower than the height of the barrier $\varphi=\left(U-\varepsilon_{F}\right), S_{b}$ can be expanded in powers of $V$ :

$$
S_{b}=q_{0}(b-c)\left\{1-\frac{m_{0} e V}{2 q_{0}^{2}}\left(\frac{b-c}{w}\right)+\ldots\right\}
$$

which shows that $\Gamma^{R}(\mathbf{c}) \sim \exp \left(-S_{b} / \hbar\right)$ is an increasing function of $V$ in the vicinity of $V=0$. Hence, it leads to increase of differential conductivity $\sigma(V)=\partial I / \partial V$ under direct bias voltage, applied to the barrier from the left to the right direction, and to decrease of $\sigma(V)$ under inverse bias voltage. The physical meaning of such behavior is rather obvious. From expression for $S_{b}$ it follows that electrons tunneling under forward bias due to resonant levels lying close to $\varepsilon_{r}$ will propagate through the potential barrier with the height which is effectively less compared with that in case of inverse bias.

The expression for the paramagnetic impurity assisted current at finite voltages has the structure similar to exp. (19) with Fermi distribution factors written in accordance with the general formula (17) and integrand expression has the form given in Appendix A, where line-widths $\Gamma_{\uparrow(\downarrow)}$ have to be substituted by WKB approximation (20). In the case of magnetic impurities the above outlined mechanism of asymmetry in $I(V)$ characteristic due to the resonance levels will essentially contribute to the voltage dependence of TMR in question, and it will be discussed in the next section.

\section{Results and discussion}

In this section we consider the temperature and bias voltage dependencies of the conductances and TMR effect of the considered structures. We investigate the case of 


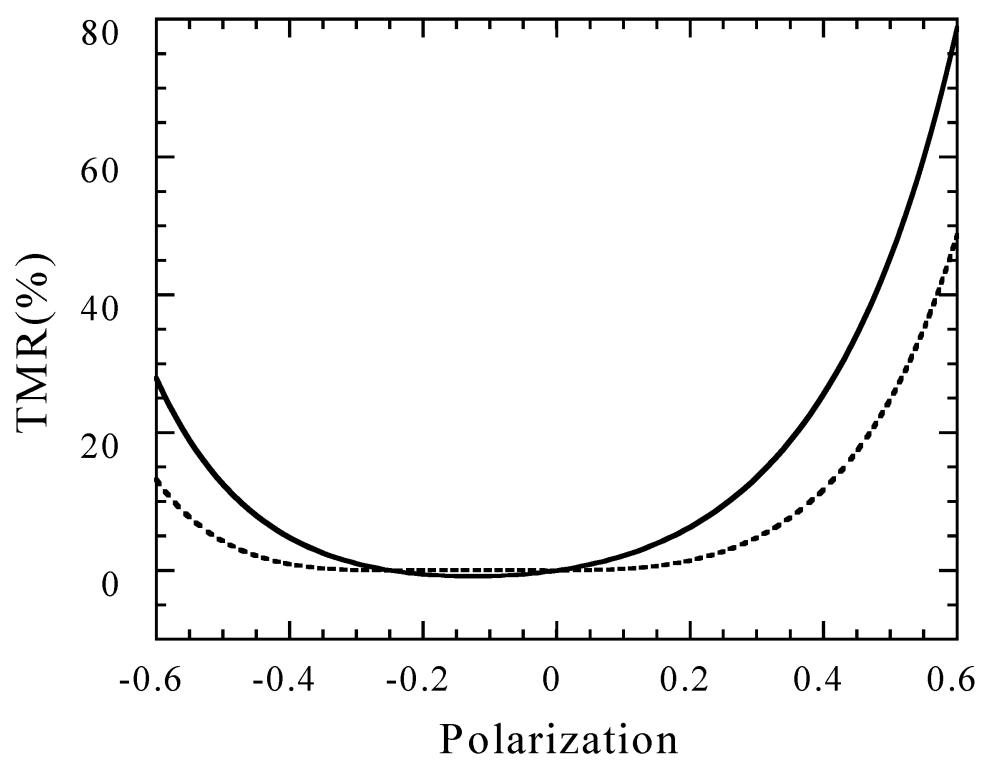

Fig. 3: Tunnel magnetoresistance at $T=0$ as a function of polarization $P=\frac{k_{\uparrow}^{F}-k_{\downarrow}^{F}}{k_{\uparrow}^{F}+k_{\downarrow}^{F}}$ under fixed $k_{\uparrow}^{F}=1.09 \stackrel{\circ}{A}^{-1}$. Other parameters are: $q_{0}=0.56 \stackrel{\circ}{A}^{-1}, m_{0}=0.4$. Solid line corresponds to the case of impurity concentration $x=8 \times 10^{-5}$, dashed line represents the case of absence of impurities.

$\mathrm{Co} / \mathrm{Al}_{2} \mathrm{O}_{3} /$ Co junction with the typical parameters that have been already mentioned in section 2: $k_{\uparrow}^{F}=1.09 \AA^{-1}, k_{\downarrow}^{F}=0.42 \AA^{-1}$ are the Fermi momenta of itinerant electrons in Co, $q_{0}=0.56 \AA^{-1}$ is the imaginary momentum in the barrier, $m_{0}=0.4$ is the effective mass in the insulator and $w=20 \AA$ is its thickness. We focus on the most interesting situation when impurities are introduced at the vicinity of the left electrode at a depth $w_{1}$ inside the insulator layer. We chose width $w_{1}=4.06 \AA$ that corresponds to two atomic monolayers. The essential parameter of the model that has to be defined is the effective molecular field $H_{z}^{\text {eff }}$ acting on impurity spins. One may suppose that it should exponentially decay in the depth of the barrier. We have, therefore, set it to $\mu_{B} H_{z}^{\text {eff }}=5 \mathrm{meV}$ $(58 \mathrm{~K})$ that is of two order less than the critical temperature in the bulk Co.

Consider, first, the case $T=0$. It is possible to estimate the concentration of impurity atoms so that its contribution to the resonance conductivity is comparable with the ordinary tunnel conductance of the system. One can write that the impurity density of states (see exp.(11)) $\nu\left(\varepsilon_{F}\right)=\frac{N_{i}}{S w_{1}(\Delta \varepsilon)}$, where $N_{i}$ is a total number of impurities and $\Delta \varepsilon$ is the width of its energy distribution. On the other hand $N_{i}=x N$ and $N=S w_{1} / a_{0}^{3}$ where $N$ is the total number of atoms in the layer which contains the impurities, $x$ is the local concentration of impurities in this volume and $a_{0}$ is the lattice constant. This yields $\nu\left(\varepsilon_{F}\right)=x\left(\frac{w_{1}}{a_{0}}\right) \frac{1}{a_{0}^{2} \Delta \varepsilon}$. We introduce the characteristic concentration $x_{0}$ defined so that in the case of nonmagnetic impurity, the impurity conductance (11) is equal to the tunnel conductance (10) of spin $\uparrow$ channel in the parallel magnetic configuration of the 
ferromagnetic layers. Such definition leads to

$$
x_{0} \simeq \frac{\Delta \varepsilon}{\pi^{2}}\left(\frac{2 m_{0} a_{0}^{2}}{\hbar^{2}}\right)\left(\frac{w}{w-w_{1}}\right)\left(\frac{a_{0}}{w_{1}}\right) \frac{k_{\uparrow}^{F} q_{0} m_{0}}{k_{\uparrow}^{F^{2}} m_{0}^{2}+q_{0}^{2}} \frac{\left(q_{0} w_{1}\right) \exp \left(-2 q_{0} w_{1}\right)}{1-\exp \left(-2 q_{0} w_{1}\right)}
$$

If we choose $\Delta \varepsilon=0.2 \mathrm{eV}$, then $x_{0}=6.5 \times 10^{-5}$. The conductance of the system at $T=0$ can be extract from the general expression (14). We suppose that for both parallel and antiparallel configurations the left electrode has "up" magnetization and, hence, $H_{z}^{\text {eff }}$ is positive in both cases. At zero temperature, all spin-flip processes are frozen and due to the above assumption, only the configuration of impurity spin with $m_{s}=1$ is possible. As a result, only two resonance channels from many possible ones have nonzero contribution to conductivity, namely $\left|\uparrow, m_{s}=1\right\rangle \leftrightarrow\left|\uparrow, m_{s}=1\right\rangle$ and $\left|\downarrow, m_{s}=1\right\rangle \leftrightarrow \mid \downarrow, m_{s}=$ 1) with $m_{j}=3 / 2$ and $1 / 2$ respectively. From exp. (A1) (see Appendix A) it follows that the channel with $m_{j}=3 / 2$ gives the main contribution into the conductivity at low temperatures and ${ }^{P} \sigma_{3 / 2}^{\uparrow \uparrow} \sim \Gamma_{\uparrow}$ and ${ }^{A P} \sigma_{3 / 2}^{\uparrow \uparrow} \sim \Gamma_{\downarrow}$. So these contributions depend on the mutual orientation of the magnetization of the ferromagnetic layers, therefore they increase the total amplitude of the TMR. The total expression for $T M R=\frac{\sigma^{P}-\sigma^{A P}}{\sigma^{A P}}$ including all possible channels may be written as

$$
T M R=\frac{\left(\Gamma_{\uparrow}-\Gamma_{\downarrow}\right)\left(\Gamma_{\uparrow}-\Gamma_{\downarrow}+\frac{x}{x_{0}} \Gamma_{\uparrow}\left(1-\frac{1}{9} \Gamma_{\downarrow} / \gamma_{\uparrow}\right)\right)}{\Gamma_{\uparrow} \Gamma_{\downarrow}\left(2+\frac{x}{x_{0}}\left(1+\frac{1}{9} \Gamma_{\uparrow} / \gamma_{\uparrow}\right)\right)}
$$

where $x$ is concentration, $x_{0}$ is defined by (21), $\gamma_{\uparrow}=\frac{2}{3} \Gamma_{\uparrow}+\frac{1}{3} \Gamma_{\downarrow}$ and $\Gamma_{\uparrow(\downarrow)}=k_{\uparrow \downarrow}^{F} q_{0}^{2} /\left(k_{\uparrow(\downarrow)}^{F 2} m_{0}^{2}+\right.$ $\left.q_{0}^{2}\right)$ are the tunneling density of states for $\uparrow(\downarrow)$ spin electrons. The dependence of TMR effect versus the polarization $P=\left(k_{\uparrow}^{F}-k_{\downarrow}^{F}\right) /\left(k_{\uparrow}^{F}+k_{\downarrow}^{F}\right)$ is shown in Fig. 3 in comparison with the non-resonant tunnel conductance at $x=0$. The total TMR amplitude is larger than the TMR due to direct tunneling, in accordance with considerations written above. For a given polarization $P=0.44$ in case of chosen parameters, the contribution of the impurity assisted tunneling leads to strong enhancement of TMR amplitude (typically by a factor 2, see Fig. 3).

We note, that in the case of nonmagnetic impurities, distributed in the vicinity of only one contact, the resonant impurity conductance $\sigma^{\text {imp }} \sim \Gamma_{\uparrow}+\Gamma_{\downarrow}$ is equal for both parallel and antiparallel configurations and, therefore, in this case the mechanism of impurity assisted tunneling is not able to enhance the TMR effect. The enhancement of TMR amplitude in the case of paramagnetic impurities is essentially due to the presence of ferromagnetic exchange coupling between the magnetization in ferromagnetic electrode and the impurity spins which tends to induce a ferromagnetic order in the plane of impurities and, as the result, leads to the preference of impurity spin to be found in the quantum state with $m_{s}=1$.

The temperature dependences of resonant conductances for parallel and antiparallel configurations in the interval from $4.2 \mathrm{~K}-300 \mathrm{~K}$ are presented in Fig. 4 . In the case of parallel alignment, $\sigma_{\text {imp }}^{P}(T)$ is nearly independent on the temperature, but in antiparallel situation there is a $50 \%$ increase of impurity conductance $\sigma_{\text {imp }}^{A P}(T)$. This originates from the thermal excitation of both spin-flip and spin-conserving processes which are frozen at zero temperature. For AP configuration the process $\left|\uparrow, m_{s}=0\right\rangle \rightarrow\left|\downarrow, m_{s}=1\right\rangle$ was forbidden at $0^{\circ} \mathrm{K}$ but now it is allowed and gives a large contribution into the current as it is proportional to the product of the largest density of states $\Gamma_{\uparrow} \Gamma_{\uparrow}$. As a consequence, the TMR effect decreases with the increase of the temperature. 


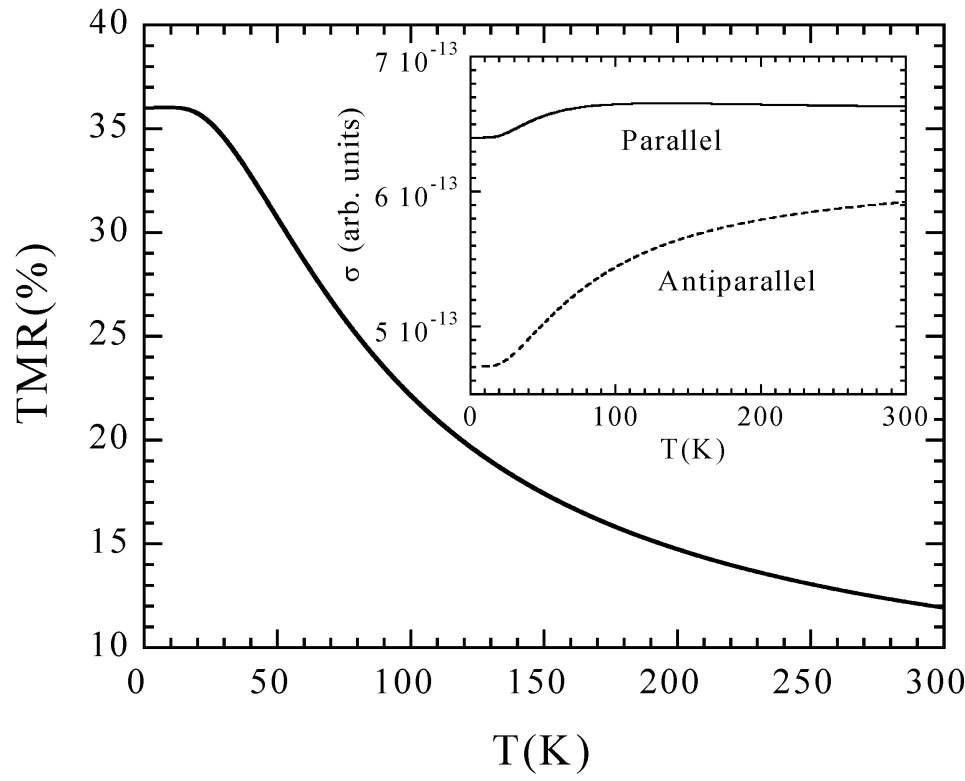

Fig. 4: Tunnel magnetoresistance as a function of the temperature. (On the insertion: conductance $\sigma$ for the parallel and antiparallel configurations.) Parameters are: $k_{\uparrow}^{F}=1.09 \AA^{-1}$, $k_{\downarrow}^{F}=0.42 \stackrel{\circ}{\AA^{-1}}, q_{0}=0.56 \stackrel{\circ}{A^{-1}}, m_{0}=0.56$. Concentration of impurities $x=8 \times 10^{-5}$.

We have also calculated the dependence of the differential conductances on bias voltage according to (18) and (19). These dependences at $T=4.2 \mathrm{~K}$ and $T=77 \mathrm{~K}$ are presented in Fig. 5. A new effect is predicted: the voltage dependence of the conductance in the antiparallel alignment of magnetization in ferromagnetic layers is asymmetric under forward and inverse bias voltage when the paramagnetic impurities inside the insulator layer are distributed close to only one of the interfaces and are bound by exchange interaction with magnetization of the nearest ferromagnetic layer.

One can distinguish two different mechanisms that give rise to the presented asymmetrical behavior with respect to inversion of bias voltage. The first one, which we refer as zero bias anomaly, manifests itself at low voltages of the order of $10 \mathrm{mV}$ (for particular chosen parameters in our model) and is strongly pronounced only at low temperatures (see Fig. 5, the case of $T=4.2 \mathrm{~K}$ ). It originates from the excitations of spin-flip processes on the impurity centers. One may look at the general expression (18) and consider the case of low temperature. An electron undergoing spin-flip scattering, may transfer an amount of energy $\omega_{0}=2 \mu_{B} H_{z}^{\text {eff }}$ to the impurity spin thus exiting it at an higher energy level or on the contrary may acquire this quantum of energy from it. The latter process is impossible at low temperature. The former one is possible only if an electron moving, say, from the left contact possesses an excess energy of at least $\omega_{0}$ with respect to Fermi level in the right contact. The only one process that contributes to this anomaly at low temperature is $\phi_{1 / 2}^{\downarrow} \rightarrow \phi_{1 / 2}^{\uparrow}$ (see exp. 15). For antiparallel alignment of the magnetization its quantum mechanical probability is proportional to ${ }^{A P} \sigma_{1 / 2}^{\downarrow \uparrow} \sim \frac{2}{9} \Gamma_{\downarrow}^{2} / \gamma_{\uparrow}$ for electrons moving from the left ferromagnetic layer into the right one and is proportional to ${ }^{A P} \sigma_{1 / 2}^{\uparrow \downarrow} \sim \frac{2}{9} \Gamma_{\uparrow}^{2} / \gamma_{\uparrow}$ in the case of electrons moving from the right to the left. For parallel configuration of magnetizations these probabilities are equal in both directions and are proportional to ${ }^{P} \sigma_{1 / 2}^{\downarrow \uparrow}={ }^{P} \sigma_{1 / 2}^{\uparrow \downarrow} \sim \frac{2}{9} \Gamma_{\downarrow} \Gamma_{\uparrow} / \gamma_{\uparrow}$. As a result, zero bias anomaly at $T=4.2 \mathrm{~K}$ looks asymmet- 


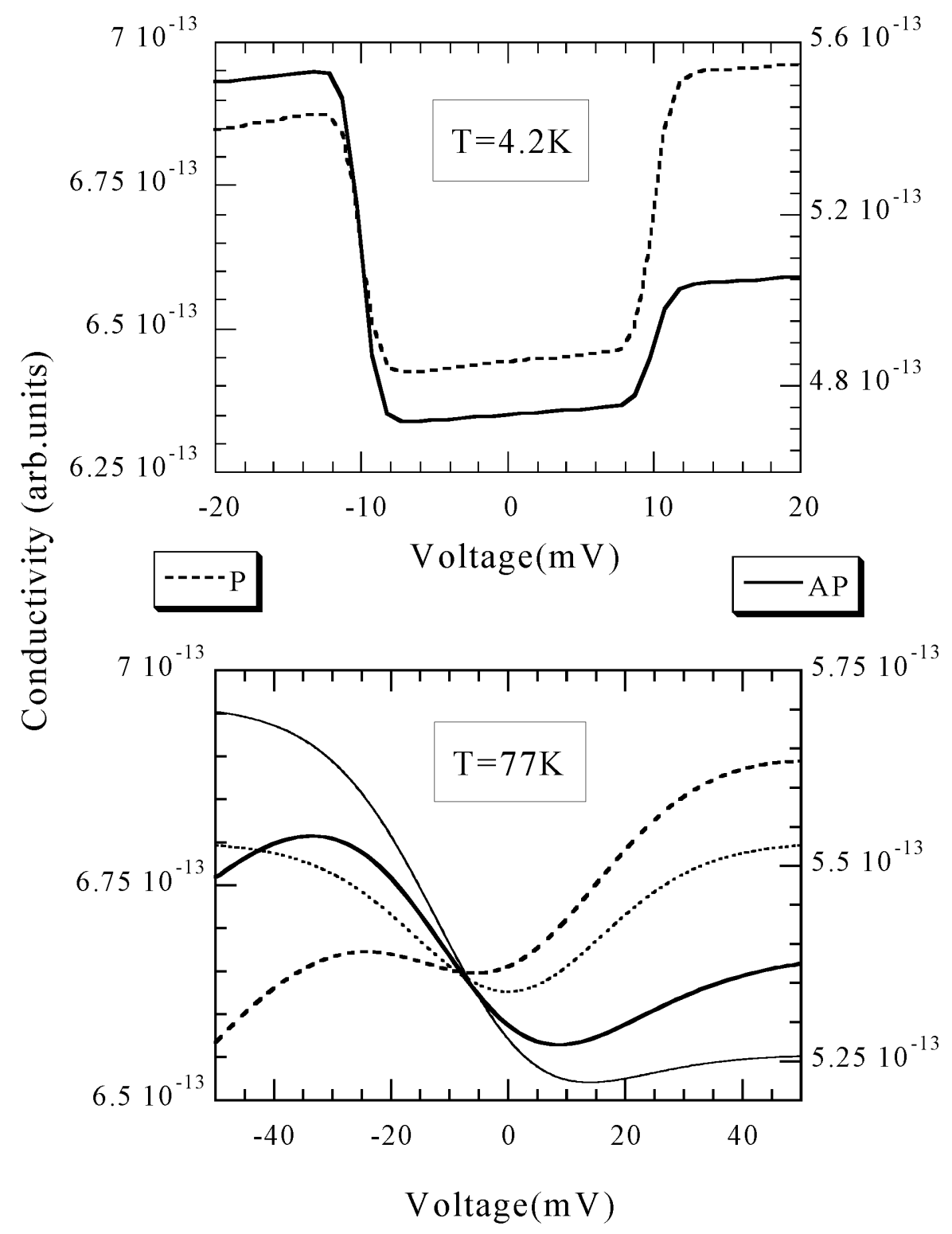

Fig. 5: Differential conductance as a function of the bias voltage at $T=4.2 \mathrm{~K}$ and $77 \mathrm{~K}$. Parameters are: $k_{\uparrow}^{F}=1.09 \stackrel{\circ}{A}-1, k_{\downarrow}^{F}=0.42 \stackrel{\circ}{A}^{-1}, q_{0}=0.56 \stackrel{\circ}{A^{-1}}, m_{0}=0.4$. Thick dashed and solid lines correspond to the conductance in the parallel and antiparallel configurations respectively, calculated in the WKB approximation. For the comparison, the same dependences at $T=77 \mathrm{~K}$, calculated by approximate formulae, are indicated by thin lines. Concentration of impurities $x=8 \times 10^{-5}$. 


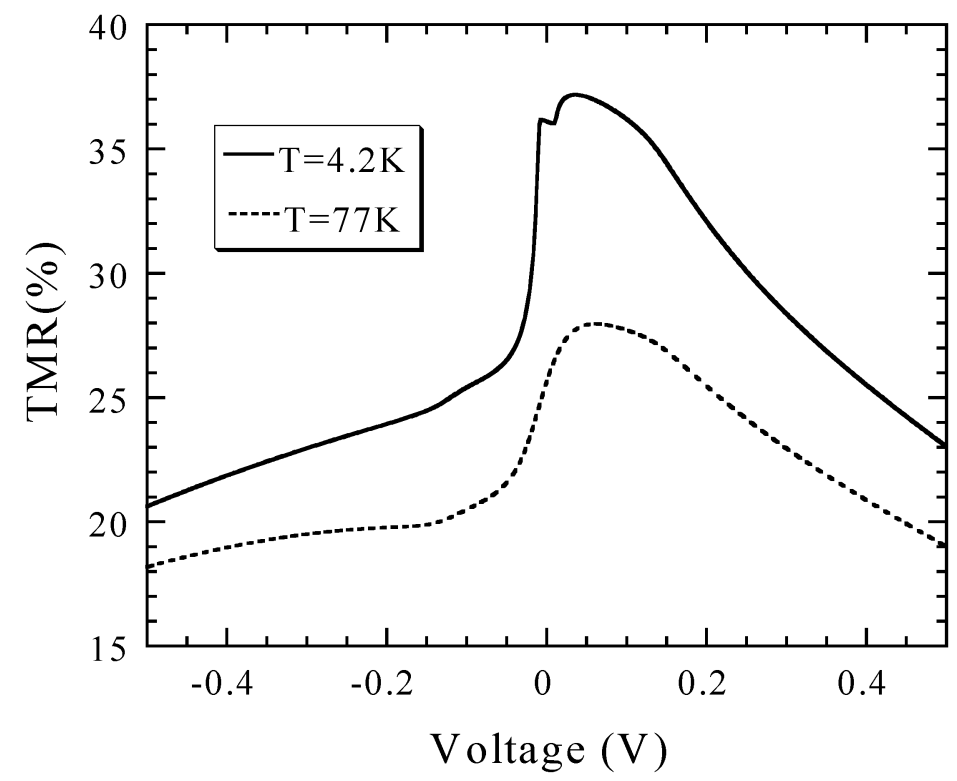

Fig. 6: Tunnel magnetoresistance as a function of the bias voltage: solid line $-T=4.2 \mathrm{~K}$, dashed line - $T=77 \mathrm{~K}$. Parameters are: $k_{\uparrow}^{F}=1.09 \stackrel{\circ}{A}^{-1}, k_{\downarrow}^{F}=0.42 \stackrel{\circ}{A^{-1}}, q_{0}=0.56 \AA^{-1}$, $m_{0}=0.4$. Concentration of impurities $x=8 \times 10^{-5}$.

rically in the case of antiparralel configuration and is symmetrical in the case of parallel alignment of magnetizations.

The differential conductances as a functions of the bias voltage at $T=77 \mathrm{~K}$ have been calculated using two different approximations. Thick dashed and solid lines correspond to the conductances in the parallel and antiparallel configurations, respectively, that have been calculated by means of WKB approximation in accordance with expressions (17) and (19). For the sake of comparison, the same dependences, indicated by thin lines, have been calculated with the use approximate formulae (18), where the dependence of $\hat{t}$-matrix elements on the applied voltage has been neglected. In course of this, the latter curves demonstrate the only zero bias anomaly discussed above, which is substantially smoothed, compared with the case of $T=4.2 \mathrm{~K}$. On the contrary, the WKB scheme of calculation takes into account the variation of the potential profile inside the insulating barrier under applied bias voltage. In view of this, the differential conductances calculated by this scheme exhibit the tendency to increase at the direct bias voltage and to decrease at the reverse one. As it was shown above (see section 2.4), this behavior originates from the shift of the resonant levels inside the insulator due to externally applied electric field. This second mechanism in the origin of non-linear voltage dependence of impurity assisted conductance does not relate with the excitation of spin-flip processes. It becomes apparent at the voltages of the order of $50 \mathrm{mV}$ and leads to the asymmetric voltage bias dependences in both cases of parallel and antiparallel configurations.

Finally, the TMR amplitude as a function of the bias voltage is shown in Fig. 6 for the broad range of applied voltage. Its non-linear and asymmetric behavior in the range of $0.2 \mathrm{~V}$ originates from the asymmetry of the shifts of resonant impurity levels with respect to forward and inverse bias. The low bias voltage anomaly at $10 \mathrm{mV}$ is also strongly pronounced at the curve corresponding to $4.2 \mathrm{~K}$. The relative contribution of the impurity assisted conductance to the total current of electrons is diminished after the 
value of applied bias voltage exceeds the half width of impurity band $\Delta \varepsilon / 2=0.1 \mathrm{eV}$. Therefore, the TMR amplitude drops to value $\simeq 20 \%$ at $0.5 \mathrm{~V}$ corresponding primary to the pure tunnel conductance.

\section{Acknowledgments}

A. Vedyayev and D. Bagrets are grateful to CEA/Grenoble/DRFMC/SP2M/NM for hospitality. This work was partially supported by Russian Foundation for Basic Research (grant No. 98-02-16806).

\section{Appendix A}

Let $w_{1}=c-a$ and $w_{2}=b-c$ be the position of impurity with respect to the left and right interfaces, respectively, and $w=b-a$ be the width of the insulator layer. We introduce tunneling densities of states for spin $\uparrow(\downarrow)$ electrons $\Gamma_{\uparrow(\downarrow)}=k_{\uparrow \downarrow}^{F} q_{0}^{2} /\left(k_{\uparrow(\downarrow)}^{F} m_{0}^{2}+q_{0}^{2}\right)$ and denote $\gamma_{\uparrow}=\frac{2}{3} \Gamma_{\uparrow}+\frac{1}{3} \Gamma_{\downarrow}, \gamma_{\downarrow}=\frac{1}{3} \Gamma_{\uparrow}+\frac{2}{3} \Gamma_{\downarrow}$. Then, the position-dependent quantum mechanical probabilities $\sigma_{m_{j}}^{\mu \rho}(c)$ can be found as follows.

(a) In case of parallel configuration:

$$
\begin{aligned}
& \sigma_{\frac{3}{2}\left(-\frac{3}{2}\right)}^{\uparrow \uparrow(\downarrow \downarrow)}(c)=\frac{\Gamma_{\uparrow(\downarrow)}^{2} e^{-2 q_{0} w} /\left(w_{1} w_{2}\right)}{\Gamma_{\uparrow(\downarrow)} \frac{e^{-2 q_{0} w_{1}}}{w_{1}}+\Gamma_{\uparrow(\downarrow)} \frac{e^{-2 q_{0} w_{2}}}{w_{2}}}, \\
& \underset{\frac{1}{2}\left(-\frac{1}{2}\right)}{\sigma_{\uparrow}(\downarrow \downarrow)}(c)=\frac{4}{9} \frac{\Gamma_{\uparrow(\downarrow)}^{2} e^{-2 q_{0} w} /\left(w_{1} w_{2}\right)}{\gamma_{\uparrow(\downarrow)} \frac{e^{-2 q_{0} w_{1}}}{w_{1}}+\gamma_{\uparrow(\downarrow)} \frac{e^{-2 q_{0} w_{2}}}{w_{2}}},
\end{aligned}
$$

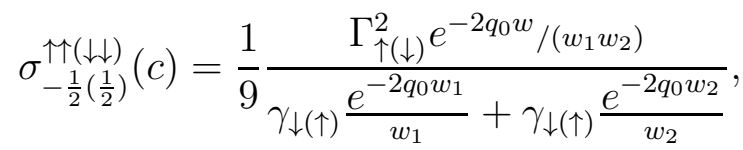

$$
\begin{aligned}
& \sigma_{\frac{1}{2}}^{\downarrow \uparrow}(c)=\sigma_{\frac{1}{2}}^{\uparrow \downarrow}(c)=\frac{2}{9} \frac{\Gamma_{\uparrow} \Gamma_{\downarrow} e^{-2 q_{0} w} /\left(w_{1} w_{2}\right)}{\gamma_{\uparrow} \frac{e^{-2 q_{0} w_{1}}}{w_{1}}+\gamma_{\uparrow} \frac{e^{-2 q_{0} w_{2}}}{w_{2}}}, \\
& \sigma_{-\frac{1}{2}}^{\uparrow \downarrow}(c)=\sigma_{-\frac{1}{2}}^{\downarrow \uparrow}(c)=\frac{2}{9} \frac{\Gamma_{\uparrow} \Gamma_{\downarrow} e^{-2 q_{0} w} /\left(w_{1} w_{2}\right)}{\gamma_{\downarrow} \frac{e^{-2 q_{0} w_{1}}}{w_{1}}+\gamma_{\downarrow} \frac{e^{-2 q_{0} w_{2}}}{w_{2}}} ;
\end{aligned}
$$

(b) In case of antiparallel configuration:

$$
\begin{aligned}
\sigma_{\frac{3}{2}\left(-\frac{3}{2}\right)}^{\uparrow \uparrow(\downarrow \downarrow)}(c) & =\frac{\Gamma_{\uparrow} \Gamma_{\downarrow} e^{-2 q_{0} w} /\left(w_{1} w_{2}\right)}{\Gamma_{\uparrow(\downarrow)} \frac{e^{-2 q_{0} w_{1}}}{w_{1}}+\Gamma_{\downarrow(\uparrow)} \frac{e^{-2 q_{0} w_{2}}}{w_{2}}}, \\
\sigma_{\frac{1}{2}\left(-\frac{1}{2}\right)}^{\uparrow \uparrow(\downarrow \downarrow)}(z) & =\frac{4}{9} \frac{\Gamma_{\uparrow} \Gamma_{\downarrow} e^{-2 q_{0} w} /\left(w_{1} w_{2}\right)}{\gamma_{\uparrow(\downarrow)} \frac{e^{-2 q_{0} w_{1}}}{w_{1}}+\gamma_{\downarrow(\uparrow)} \frac{e^{-2 q_{0} w_{2}}}{w_{2}}},
\end{aligned}
$$




$$
\begin{gathered}
\sigma_{-\frac{1}{2}\left(\frac{1}{2}\right)}^{\uparrow \uparrow(\downarrow \downarrow)}(z)=\frac{1}{9} \frac{\Gamma_{\uparrow} \Gamma_{\downarrow} e^{-2 q_{0} w} /\left(w_{1} w_{2}\right)}{\gamma_{\downarrow(\uparrow)} \frac{e^{-2 q_{0} w_{1}}}{w_{1}}+\gamma_{\uparrow(\downarrow)} \frac{e^{-2 q_{0} w_{2}}}{w_{2}}}, \\
\sigma_{\frac{1}{2}}^{\uparrow \downarrow(\downarrow \uparrow)}(z)=\frac{2}{9} \frac{\Gamma_{\uparrow(\downarrow)}^{2} e^{-2 q_{0} w_{1}} /\left(w_{1} w_{2}\right)}{\gamma_{\uparrow} \frac{e^{-2 q_{0} w_{1}}}{w_{1}}+\gamma_{\downarrow} \frac{e^{-2 q_{0} w_{2}}}{w_{2}}}, \\
\sigma_{-\frac{1}{2}}^{\tau_{\downarrow}(\downarrow \uparrow)}(z)=\frac{2}{9} \frac{\Gamma_{\uparrow(\downarrow)}^{2} e^{-2 q_{0} w} /\left(w_{1} w_{2}\right)}{\gamma_{\downarrow} \frac{e^{-2 q_{0} w_{1}}}{w_{1}}+\gamma_{\uparrow} \frac{e^{-2 q_{0} w_{2}}}{w_{2}}} .
\end{gathered}
$$

The statistical probabilities $P_{j}^{\mu \rho}(h)$ are independent of the configuration of the system. We denote $h=\mu_{B} H_{z}^{\text {eff }} / k T$ and $Z=2 \cosh (2 h)+1$, then

$$
\begin{gathered}
P_{\frac{3}{2}}^{\uparrow \uparrow}(h)=P_{\frac{1}{2}}^{\downarrow \downarrow}(h)=Z^{-1} e^{2 h}, \quad P_{\frac{1}{2}}^{\uparrow \uparrow}(h)=P_{-\frac{1}{2}}^{\downarrow \downarrow}(h)=Z^{-1}, \\
P_{-\frac{1}{2}}^{\uparrow \uparrow}(h)=P_{-\frac{3}{2}}^{\downarrow \downarrow}(h)=Z^{-1} e^{-2 h}, \\
P_{\frac{1}{2}}^{\uparrow \downarrow}(h)=P_{\frac{1}{2}}^{\downarrow \uparrow}(h)=Z^{-1} \frac{h e^{h}}{\sinh (h)}, \quad P_{-\frac{1}{2}}^{\uparrow \downarrow}(h)=P_{-\frac{1}{2}}^{\downarrow \uparrow}(h)=Z^{-1} \frac{h e^{-h}}{\sinh (h)}
\end{gathered}
$$

\section{Appendix B}

The non-trivial functions $I_{m_{j}}^{\mu \rho}$ are written as follows

$$
\begin{aligned}
I_{1 / 2}^{\downarrow \uparrow}\left(V, H_{z}^{\text {eff }}\right) & =\frac{\left(e V-2 \mu_{B} H_{z}^{\text {eff }}\right)\left(e^{e V / k T}-1\right)}{\left(e^{\left(e V-2 \mu_{B} H_{z}^{\text {eff }}\right) / k T}-1\right)\left(2 \cosh \left(2 \mu_{B} H_{z}^{\text {eff }} / k T\right)+1\right)}, \\
I_{1 / 2}^{\uparrow \downarrow}\left(V, H_{z}^{\text {eff }}\right) & =\frac{\left(e V+2 \mu_{B} H_{z}^{\text {eff }}\right)\left(e^{e V / k T}-1\right) e^{2 \mu_{B} H / k T}}{\left(e^{\left(e V+2 \mu_{B} H_{z}^{\text {eff }}\right) / k T}-1\right)\left(2 \cosh \left(2 \mu_{B} H_{z}^{\text {eff }} / k T\right)+1\right)}, \\
I_{-1 / 2}^{\downarrow \uparrow}\left(V, H_{z}^{\text {eff }}\right) & =\frac{\left(e V+2 \mu_{B} H_{z}^{\text {eff }}\right)\left(e^{e V / k T}-1\right)}{\left(e^{\left(e V+2 \mu_{B} H_{z}^{\text {eff }}\right) / k T}-1\right)\left(2 \cosh \left(2 \mu_{B} H_{z}^{\text {eff }} / k T\right)+1\right)}, \\
I_{-1 / 2}^{\uparrow \downarrow}\left(V, H_{z}^{\text {eff }}\right) & =\frac{\left(e V-2 \mu_{B} H_{z}^{\text {eff }}\right)\left(e^{e V / k T}-1\right) e^{-2 \mu_{B} H / k T}}{\left(e^{\left(e V-2 \mu_{B} H_{z}^{\text {eff }}\right) / k T}-1\right)\left(2 \cosh \left(2 \mu_{B} H_{z}^{\text {eff }} / k T\right)+1\right)} .
\end{aligned}
$$

All the other ones, not written above, are equal to $\mathrm{eV}$.

\section{References}

[1] J. S. Moodera, L. R. Kinder, T. M. Wong, and R. Meservey, Phys. Rev. Lett., 74, 3273 (1995)

[2] T. Miyazaki and N. Tezuka, J. Magn. Magn. Mater., 139, L231 (1995)

[3] W. J. Gallagher, S. S. S. Parkin, Y. Lu, X. P. Bian, A. Marley et al., J. Appl. Phys., 81, 3741 (1997)

[4] J. C. Słonczewski, Phys. Rev. B, 39, 6995 (1989)

[5] A. Vedyayev, N. Ryzhanova, C. Lacroix, L. Giacomoni, and B. Dieny, Europhys. Lett., 39, $219(1997)$ 
[6] A. Vedyayev, N. Ryzhanova, R. Vlutters, and B. Dieny, Europhys. Lett., 46, 808 (1999)

[7] I. M. Lifshitz and V. Ya. Kirpichenkov, Zh. Éksp. Teor. Fiz., 77, 989 (1979) [Sov. Phys. JETP, 50, 499 (1979)]

[8] A. I. Larkin and K. A. Matveev, Zh. Éksp. Teor. Fiz., 93, 1030 (1987) [Sov. Phys. JETP, 66, $580(1987)]$

[9] A. M. Bratkovsky, Pis'ma Zh. Éksp. Teor. Fiz., 65 (5), 430 (1997) [JETP Letters, 65 (5), $452(1997)]$

[10] E. Yu. Tsymbal and D. G. Pettifor, Phys. Rev. B, 58, 432 (1998)

[11] H. Itoh, T. Kumazaki, J. Inoe, and S. Maekawa, Japan J. Appl. Phys., 37, 554 (1998)

[12] F. Guinea, Phys. Rev. B, 58, 9212 (1998)

[13] R. Jansen and J. C. Lodder, Phys. Rev. B, 615860 (2000). In this work the spin-dependent polarized densities of resonant states $\nu_{\uparrow}$ and $\nu_{\downarrow}$ have been introduced into the consideration. However, no physical mechanism underlying these phenomenological quantities has been proposed by the authors. We may note, that for the first time the term "spin-polarized resonant tunneling" was proposed in Ref. [14]. In the latter work the tunneling through the double-barrier tunnel structure has been investigated. The case of a triple-barrier junction was later on investigated in [19]. In these structures spin-polarized resonant density of states exists in fact, but it arises due to the formation of so-called quantum-well states.

[14] Xiangdong Zhang, Bo-Zhang Li, Gang Sun, Fu-Cho Pu, Phys. Rev. B. 565484 (1997)

[15] S. Zhang, P. Levy, A. C. Marley, and S. S. P. Parkin, Phys. Rev. Lett., 793744 (1997)

[16] R. Kubo, M. Toda and N. Hashitsume. Statistical Physics II. Nonequilibrium Statistical Mechanics. (Springer, Berlin, 1985)

[17] E. M. Lifshitz and L. P. Pitaevskii. Physical Kinetics (Course of Theoretical Physics, Vol. 10). (Oxford: Pergamon Press, 1981)

[18] L. D. Landau and I. M. Lifshitz. Quantum Mechanics (Course of Theoretical Physics, Vol. 3). (Oxford: Pergamon Press, 1977)

[19] A. Vedyayev, N. Ryzhanova, R. Vlutters, B. Dieny and N. Strelkov, J.Phys.: Condens. Matter 12, 1797 (2000) 